\title{
19. European Congress for Trauma and Emergency
}

\section{Dr. med. Thomas S. Müller}

Chefarzt Zentrale Notfallstation, Stv. Leiter Unfallchirurgie, Kantonsspital Graubünden, Chur.

Präsident der Schweizerischen Gesellschaft für Traumatologie und Versicherungsmedizin (SGTV)

Anfangs Mai war es wieder soweit. Unfall- und Notfallchirurgen, Anästhesisten und Intensivmediziner aus ganz Europa mit den (Stamm-)Gästen aus Amerika und Japan fanden sich zum Jahreskongress der European Society for Trauma and Emergency Surgery, ESTES, in Valencia ein. Im vom berühmten Architekten Norman Foster geplanten und 1998 eröffneten Kongressbau trafen sich über 1200 Teilnehmer: Neben mehr als 150 spanischen Kollegen und annähernd so vielen Kollegen aus den Niederlanden, reisten eben auch 155 Ärzte aus Japan und den USA nach Valencia. Die Schweiz war mit 65 Medizinern und unzähligen wissenschaftlichen Kongressbeiträgen sehr gut vertreten: Man sah und hörte die grosse, sehr aktive Delegation aus Chur (viele Posters und Vorträge), Bern und Zürich. Und auch eine kleine Gruppe der SUVA war da, Zeichen dafür, dass sich auch die nationale Versicherungsmedizin für diese europäische Szene interessiert.

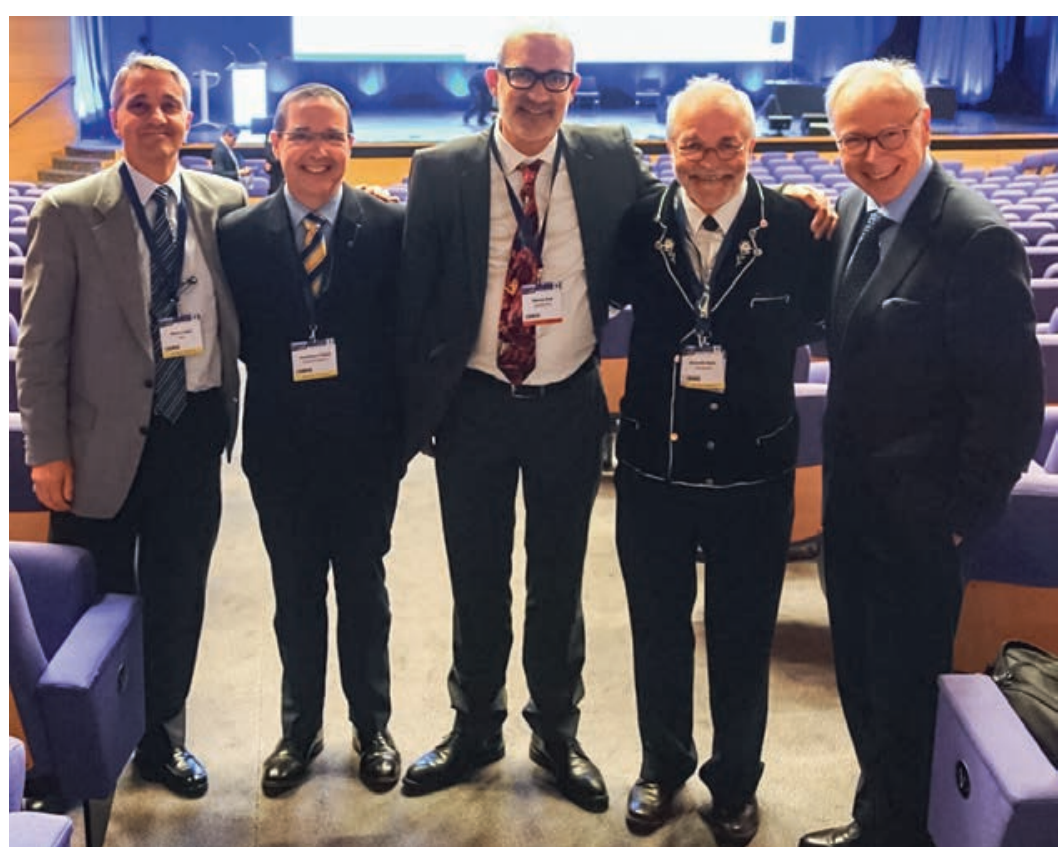

Vorstandsmitglieder der ESTES mit dem neuen Ehrenmitglied Dominik Heim. (Von links): Vorsteher des ESTES Advisory Council, Mauro Zago, Italien, Past-Präsident Jonathan Tilsed, England, Präsident Marius Keel, Schweiz, Ehrenmitglied Dominik Heim, und Präsident Elect Christoph Josten, Deutschland.

\section{Starre Kongressstrukturen bewusst aufgebrochen}

Es war ein äusserst gelungener Kongress unter der Leitung von Dr. Isidro Martinez Casas, dem nationalen Delegierten Spaniens, mit einer magistralen Führung des ESTES-Präsidenten 2017-2018, Prof. Dr. med. Marius Keel, Unfallchirurg und Orthopäde am Traumazentrum Hirslanden Zürich und Senior Consultant am Inselspital Bern. Man spürte die Handschrift der beiden innovativen und engagierten Magistraten ubiquitär an diesem Kongress. Die starren Kongressstrukturen wurden bewusst aufgebrochen und neue Formate eingeführt. Die fachlich hochstehenden Referate wurden aufgelockert durch battle-sessions, poster walks, Prosand-Cons-Debatten und den (üblichen) Guest Symposia und Industry Supported Sessions. Das Ganze komprimiert auf zweieinhalb Tage.

Die Industriebeteiligung war (auch dank des intensiven Lobbyings des ESTES-Präsidenten) wieder angestiegen. Man spürte aber in diesem Sektor die seit einigen Jahren zunehmenden Sparbemühungen der Industrie und die immer restriktiveren gesetzlichen Auflagen: Die vielen kleinen Aufmerksamkeiten an den Ständen sind verschwunden, Kugelschreiber waren selten (wohl deshalb nahm eine Kongressteilnehmerin grad deren 5!), wenig Schoggi und kein Kaffee - eine eigentliche «désertification industrielle» (von désert) hat da eingesetzt!

\section{Schweizer Vertretung}

Zum ersten Mal nach der Gründung wurde in der jetzt 12-jährigen Geschichte der ESTES diese Gesellschaft (Prof. Otmar Trentz war zusammen mit Prof. Sten Lennquist Gründungspräsident) von einem Schweizer, von Prof. Dr. med. Marius Keel, präsidiert. Und mit der Ernennung von PD Dr. med. Dominik Heim zum Ehrenmitglied der ESTES im Rahmen der Eröffnungsfeier, wurde die Schweiz gleich zweifach geehrt. Dominik Heim, 2014 als langjähriger Chirurgie Chefarzt des Spital Frutigen pensioniert, erfuhr damit - wie schon sein inzwischen verstorbener Vater, Urs Heim, am Kongress 


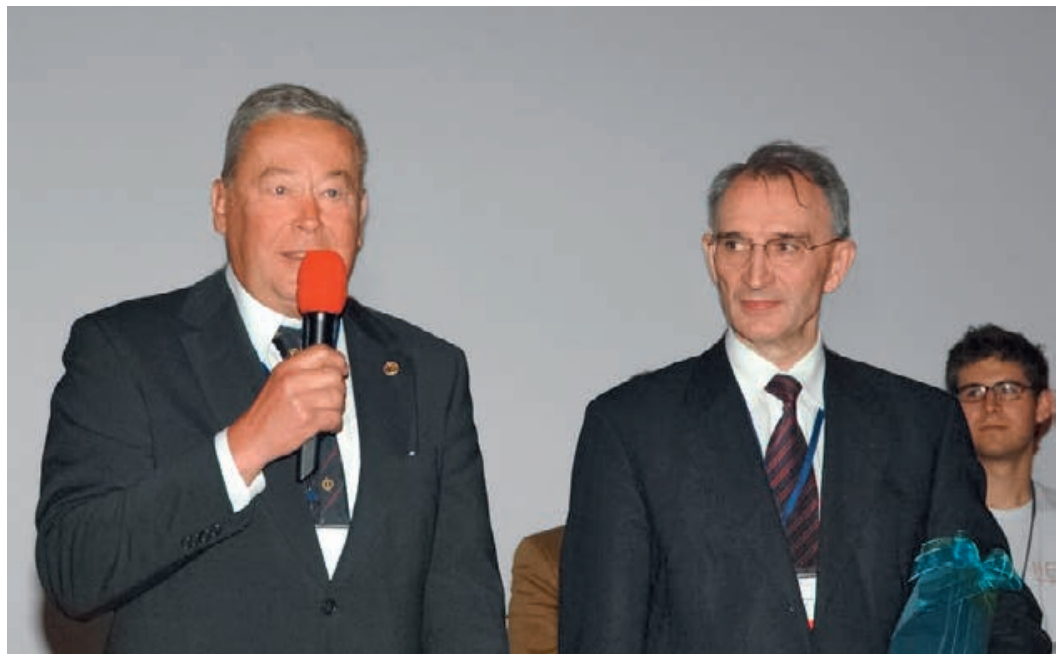

Die beiden Gründerpräsidenten, Sten Lennquist (links) und Otmar Trentz, 2007 in Graz.

2012 in Basel [1] - die höchste Ehre dieser europäischen Gesellschaft für seine Verdienste als nationaler Delegierter der Schweizerischen Gesellschaft für Traumatologie und Versicherungsmedizin, SGTV, zugunsten der ESTES (Abb. 1). Für die Schweizerischen Traumatologen und Notfallchirurgen ist es sehr erfreulich, dass mit Marius Keel und Dominik Heim zwei ehemalige SGTV-Präsidenten die Schweiz im Rahmen des ECTES 2018 in Valencia so ehrenvoll vertraten.

\section{Notfallpatienten ganzheitlich betrachten}

Und wie war das nun mit der Telefonnummer in Europa? ESTES entstand 2007 in Graz durch die Fusion der European Trauma Society (ETS) und der European Association for Trauma and Emergency Surgery (EATES) auf Bemühen der beiden damaligen Präsidenten Prof. Sten Lennquist, Stockholm, und Prof. Otmar Trentz, ehemaliger Ordinarius für Unfallchirurgie des Universitätsspitals Zürich (Abb.2). Man wusste, dass die Kräfte zum Erhalt der Allgemeinchirurgie mit Traumatologie und Notfallchirurgie gebündelt werden mussten. Das äusserst breite fachliche Spektrum wird heute durch fünf ESTES-Sektionen gezielt gestärkt. $D i-$ saster and Military Surgery, Emergency Surgery, Polytrauma, Skeletal Trauma and Sports Medicine und Visceral Trauma. In dieser europäischen Dachgesellschaft sind praktisch alle nationalen Trauma- und notfallchirurgischen Gesellschaften durch ihre nationalen Delegierten vertreten. Diese Delegierten treffen sich zusätzlich zu Skype-Meetings - zwei Mal im Jahr in einem Mitgliedland, um im ESTES advisory council (EAC) ihre nationalen Anliegen und ihren Input in die europäische Dachgesellschaft einzubringen. Das letzte EACMeeting fand in Zürich im letzten September statt. So entstanden wichtige Workshops und Kurse, aber auch europäische Empfehlungen und Leitlinien mit dem Ziel, einen europäischen Fachkonsens in zentralen notfallchirurgischen Fragen zu erreichen. Im Gegensatz zu andern europäischen Fachgesellschaften ist ESTES bestrebt, den Trauma- und Notfallpatienten als Ganzes zu verstehen und ihn nicht auf einzelne System zu reduzieren. Und so gilt es eben auch, die Aussage des ehemaligen ESTES-Präsidenten, Ari Leppaniemi, Helsinki, zu verstehen, dass ESTES die Telefon Nr.1 in Europa für den holistischen approach zum Notfallpatienten darstellt.

Der nächste Jahreskongress der ESTES (ECTES) findet vom 5. bis zum 7. Mai 2019 in Prag statt, und wir dürfen uns bestimmt wieder auf eine grosse und vor allem wissenschaftlich sehr aktive helvetische Delegation freuen.

Literatur

1 Heim D, Babst R. Basel welcomes Europe. SAEZ 2011; 92: 41: 1573

\section{Bildnachweis}

Thomas S. Müller 